\title{
Reconstruction of 3D Whole-Body PET Data Using Blurred Anatomical Labels
}

\author{
C. Comtat ${ }^{1}$, Member IEEE, P.E. Kinahan ${ }^{1}$, Member IEEE, J.A. Fessler ${ }^{2}$, Member IEEE, \\ T. Beyer ${ }^{1}$, Student Member IEEE, D.W. Townsend ${ }^{1}$, Senior Member IEEE, M. Defrise ${ }^{3}$, \\ C. Michel ${ }^{4}$, Member IEEE \\ 'Department of Radiology, University of Pittsburgh, Pittsburgh, USA \\ ${ }^{2}$ Department of Electrical Engineering and Computer Science, University of Michigan, Ann Arbor, USA \\ ${ }^{3}$ Division of Nuclear Medicine, Vrije Universiteit Brussel, AZ-VUB, Brussels, Belgium \\ ${ }^{4}$ PET Laboratory, Université Catholique de Louvain, Louvain La Neuve, Belgium
}

\begin{abstract}
s
The diagnostic utility of whole-body PET is often limited by the high level of statistical noise in the images. An improvement in image quality can be obtained by incorporating correlated anatomical information during the reconstruction of the PET data. The combined PET/CT (SMART) scanner allows the acquisition of accurately aligned PET and CT whole-body data. We present results of incorporating aligned anatomical information from the CT during the reconstruction of 3D whole-body PET data. Wé use the FORE+PWLS method for the reconstruction and a label model to incorporate anatomical information via penalty weights. Since in practice mismatches between anatomical and functional data are unavoidable, the labels are "blurred" to reflect the uncertainty associated with the anatomical information. Results show the potential advantage of incorporating anatomical information by using a blurred labels with the penalty weights.
\end{abstract}

\section{INTRODUCTION}

The role of whole-body PET imaging in oncology research and patient care is clearly growing. The diagnostic utility of whole-body oncology, however, is often limited by the high level of statistical noise in the images. Several studies have shown advantages for using anatomical information to guide reconstruction of PET data [1]. For thorax or abdomen imaging, however, it is difficult to accurately align anatomical information with PET data. The SMART scanner [2], a combined 3D PET/CT tomograph, avoids the alignment problem by acquiring both functional (PET) and anatomical (CT) data. This allows the use of accurately aligned anatomical information in the reconstruction of whole-body PET data to potentially reduce statistical noise and improve lesion detectability.

The goal of this work was to investigate the potential gain in diagnostic utility by incorporating anatomical information derived from the $\mathrm{CT}$ images in the reconstruction of 3D wholebody PET data. The method we currently use to reduce statistical noise in the PET images is our proposed FORE+OSEM combination [3] where 3D PET data are

-Address for Correspondence: P.E. Kinahan, Department of Radiology, B938-PUH, 200 Lothrop Street, Pittsburgh, PA 15213. This work is supported by grants from the Swiss National Science Foundation and NIH grants CA74135 and CA65856. M.D. and C.M. are with the National Fund for Scientific Research (Belgium). accurately rebinned to a set of stacked $2 \mathrm{D}$ sinograms by applying the Fourier rebinning technique (FORE) [4], followed by reconstruction with the accelerated 2D orderedsubsets EM reconstruction algorithm (OSEM) [5]. To extend this FORE-rebinning methodology to include anatomical information, the approach we adopt here is to minimize a penalized weighted least-squares (PWLS) cost function given by $[6]$,

$$
\Phi(\mathbf{x})=\sum_{j}\left(\sum_{i} A_{j i} x_{i}-y_{j}\right)^{2} \sigma_{j}^{-2}+\beta U(\mathbf{x})
$$

where $U(\mathbf{x})$ is a quadratic image roughness penalty function that is modified to incorporate the anatomical information. Here, $\mathbf{x}=\left\{x_{i} \mid i=1, \ldots, n\right\}$ is a vector of the $n$ voxel values of the image, $\mathrm{y}=\left\{y_{j} \mid j=1, \ldots, m\right\}$ are the $m$ projection values, $\sigma=\left\{\sigma_{j} \mid j=1, \ldots, m\right\}$ are the standard deviations for the projection data, $\mathbf{A}=\left\{A_{j i}\right\}$ is the $m \times n$ system matrix such that the expected value for $y_{j}$ is given by $\Sigma_{i} A_{i j} x_{i}$ and the parameter $\beta$ controls the influence of the image roughness penalty term $U(\mathbf{x})$. As $\Phi(\mathbf{x})$ is a quadratic function it can be readily minimized by the SOR algorithm [6]. In our implementation the 3D PET data are first rebinned with FORE to a set of transaxial 2D sinograms to accelerate image reconstruction [7].

\section{INCLUSION OF ANATOMICAL INFORMATION}

To include anatomical information we use a quadratic roughness penalty based on a $3 \mathrm{D}$ voxel neighborhood $\left(\mathrm{N}_{3 \mathrm{D}}\right)$ consisting of the 26 closest neighbors defined by,

$$
U(\mathbf{x})=\frac{1}{2} \sum_{i} \sum_{k \in N 3 \mathrm{p}(i)} \frac{1}{2} \frac{\omega_{i k}}{d_{i k}}\left(x_{i}-x_{k}\right)^{2}
$$

where $d_{i k}$ is the Euclidean distance between pixels $i$ and $k$, and the penalty weights $\omega_{i k}$ are derived from the anatomical data by using voxel labels [8]. This method associates two quantities with an image voxel: the estimated emission density and the class of material to which it belongs (e.g. lung, bone, or soft tissue). A straightforward use of the labels is to use binary penalty weights where $\omega_{i k}$ is set to one if both pixels $i$ and $k$ belong to the same class and to zero otherwise.

The use of binary penalty weights, however, may introduce unacceptable artifacts if there are mismatches between the anatomical and functional images. Fessler et al. [9] proposed a "blurred weight" method where the binary penalty weights are blurred with a kernel whose width is related to the uncertainty associated with the anatomical information. The advantage of 
such a method is that it does not require a joint estimation of both the anatomical and functional information during the reconstruction to account for anatomical and functional mismatch.

We have developed and implemented a 3D 'blurred label' method where 3D label maps $l_{j}=\left\{l_{i j} \mid i=1, \ldots, n ; 0 \leq l_{i j} \leq 1\right\}$ are defined for each class (or label) $j$ of the $m$ classes of material. The maps are initialized with mutually exclusive binary values (only one label is associated to each voxel) according to the segmented CT image Each label map is then blurred with a 3D Gaussian kernel. The penalty weights are then given by $\omega_{i k}=\Sigma_{j} l_{i j} l_{k j}$, with the requirements that $0 \leq \omega_{i k} \leq 1$ and $\Sigma_{j} l_{i j}=1$, that is, $l_{i j}$ reflects the probability of voxel $i$ belonging to tissue class (or label) $j$.

Initial anatomical classification of the voxels can be obtained by segmenting the CT image using either thresholding or statistical methods. For the studies reported here, the voxel labeling was either pre-determined or accomplished with simple thresholding.

In this paper we use simulations to investigate the utility of including the CT anatomical information in equation 2 by using the three types of penalty weights: (1) no labels $\left(\omega_{i k}=1\right)$, (2) binary labels ( $\omega_{i k}=0$ or 1 ), and (3) blurred labels $\left(0 \leq \omega_{i k} \leq 1\right)$.

We expect some level of mismatch between the PET and CT images due to, for example, respiration or patient movement during the scan. We therefore examine the performance of the different penalty weights for three conditions: (1) perfect positional and functional alignment, (2) positional mismatch, and (3) functional mismatch. By functional alignment we mean that changes in the PET emission distribution are matched with corresponding changes in the anatomical image. The use of simulation studies is important, as it allows us to estimate the mean and variance images from multiple independent realizations.

Functional mismatch has two variations. The first is where a change in the PET emission distribution is not matched with a corresponding change in the anatomical image. The second type of functional mismatch is the opposite situation where a uniform emission region has two or more different labeled sub-regions. In the limit of an isolated voxel having such a 'false' label, it is not regularized during the reconstruction process. For larger groups of voxels with a false label, the presence of statistical noise can lead to dramatic changes in the noise covariance structure, as we show below. Finally, in addition to the simulation studies, we also demonstrate the efficacy of the blurred labels method with experimental phantom data obtained from the SMART scanner.

\section{METHODS}

\section{A. Simulation Studies}

The simulation studies use a non Monte Carlo technique that allows us to generate multiple realizations of 3D sinogram data sets in a feasible computing time [7].

We first investigated the effect of accurate and mismatched labels in a noiseless simulation. The simulation was of a large uniform disk ( 30 pixel diam) with a smaller disk ( 2 pixel diam) in the center. The smaller disk had both functional and structural contrast. The $2 \mathrm{D}$ image was reconstructed with the following variations of the labels and penalty weights: (i) no labels, (ii) correct binary labels, (iii) correct blurred labels, (iv) shifted binary labels, and (v) shifted blurred labels. The use of false labels was reserved for the simulations that included statistical noise.

A large uniform disk (30 cm diam) with six embedded smaller disks of different sizes and contrast levels was the basis for 100 realizations with added statistical noise. Figure 1 shows the original object and the corresponding labels.

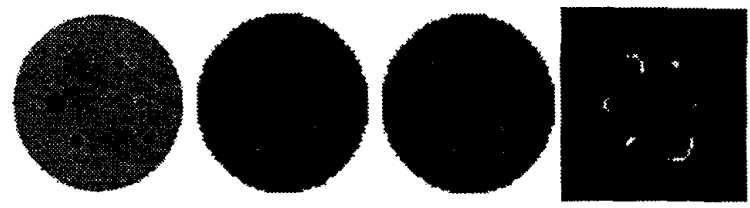

Figure 1. Emission distribution and label maps used for simulation studies. From left to right: emission distribution (showing contrast varies with diameter), original label map, shifted label map, and subtraction of original and shifted labels.

The size of the contrast disks ranged from 1 to $6 \mathrm{~cm}$ in diameter and the contrast levels of the disks varied inversely with area, with the smallest disk having the maximum contrast level of 2.25 relative to the uniform disk. The statistical noise level was set to match that of the phantom experiment described below. The effects of randoms, scatter, and attenuation correction were included, with the attenuation coefficient of all objects set to that of water.

The simulations studies were performed for three variations: (1) accurate alignment, (2) labels of the six contrast disks shifted by $5 \mathrm{~mm}$ relative to the true source distribution (displacement shown in figure 1), and (3) the emission source distribution set to a uniform background, but with the (unshifted) labels still included as 'false' labels. For each of the three label variations (accurate, shifted, and false), the 100 realizations were reconstructed with five different types of penalty weights: (1) no labels, (2) binary labels, and (3-5) labels blurred with a Gaussian kernel of FWHM 0.8, 1.0, and 1.5 pixels. Finally, the images were also reconstructed with a set of image smoothness parameters $(\beta$ in equation 2$)$ that, when used with PWLS, roughly matched the range of cutoff frequencies used with FBP in clinical imaging [7].

For each of the combinations of reconstruction parameters, pixel-wise mean and standard deviation images were calculated from the 100 realizations. Additionally, the mean bias and standard deviation of the region of interest (ROIs) was calculated for each of the 6 objects The location of the ROIs was the same for all three cases of accurate labels, shifted labels, and false labels. The mean bias and standard deviation were plotted for each object as a function of the smoothness parameter, $\beta$, and for each of the five types of penalty weights listed above.

\section{B. Experimental Studies}

To evaluate the efficacy of the blurred label method in practice, we acquired CT and [18F]-water PET scans of an elliptical torso phantom in the SMART scanner. The phantom had a series of hot and cold contrast spheres, each of 
which also contained dilute iodine-based CT contrast agent to allow for easy anatomical classification. The volume of the spheres ranged from 1 to $15 \mathrm{ml}$. The CT scan was acquired in spiral mode, with an axial motion of $3 \mathrm{~mm}$ per scanner rotation, and the images were reconstructed with a $3.4 \mathrm{~mm}$ spacing. The PET scan collected $2 \times 10^{7}$ coincidences with a deadtime of $5 \%$ and random coincidence fraction of $20 \%$.
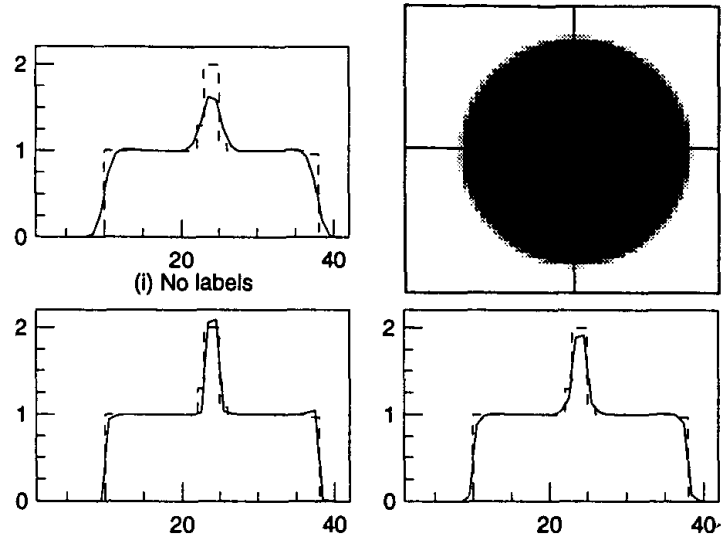

(ii) Binary labels

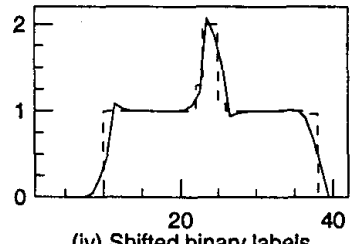

(iv) Shifted binary labels
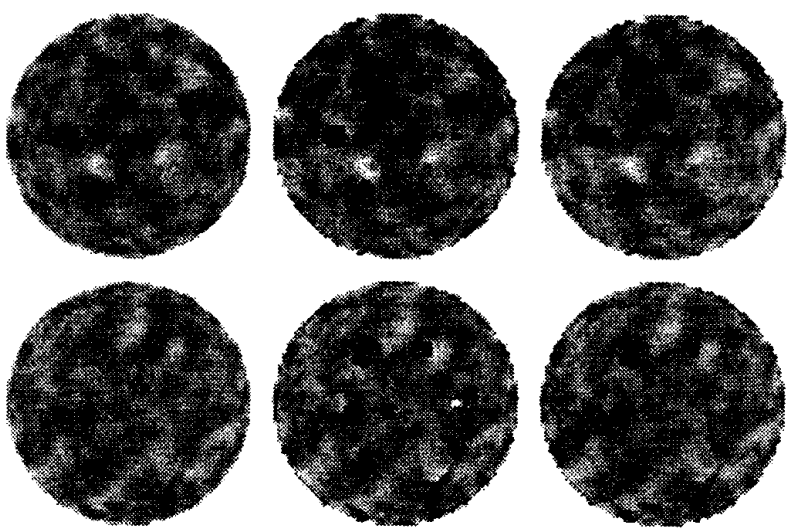

Figure 3. Two realizations from simulations with accurate labels. The top and bottom rows are independent realizations, while the columns are, from left to right: no labels, binary labels, and blurred labels.

The bias vs. standard deviation curves for all objects varied gradually according to size, and representative results are shown in figure 5 for the second to smallest and second to largest contrast objects (top two objects in figure 1 (left)). The effect of false labels is demonstrated in the two realizations shown in figure 6 and the mean and standard deviation images shown in figure 7 .
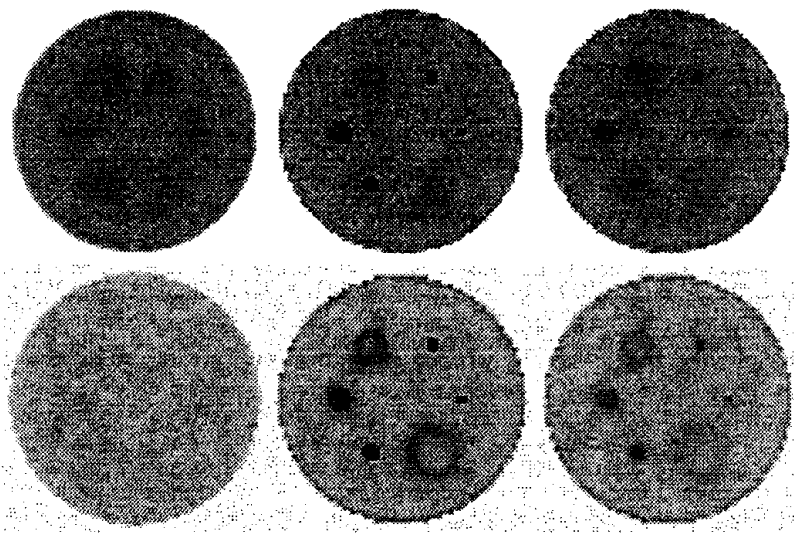

Figure 4. Mean and standard deviation images of the 100 realizations of simulations with accurate labels. Top row: mean images. Bottom row: standard deviation images. The columns correspond to figure 3 and are, from left to right: no labels, binary labels, and blurred labels.

\footnotetext{
${ }^{1}$ The same smoothness parameter $\beta$ is used for all images displayed in this section.
} 


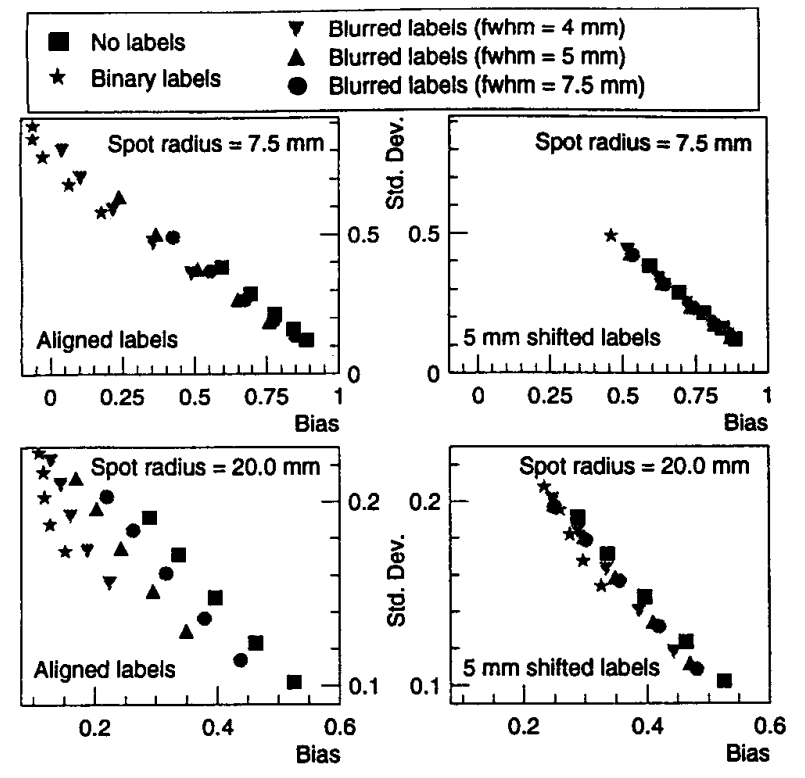

Figure 5. Bias $\left(b_{i}\right)$ vs. standard deviation $\left(s_{i}\right)$ graphs for both accurate and shifted labels for the 2nd smallest $(i=2)$ and 2 nd largest $(i=5)$ contrast objects as a function of regularization parameter and type of penalty weight.
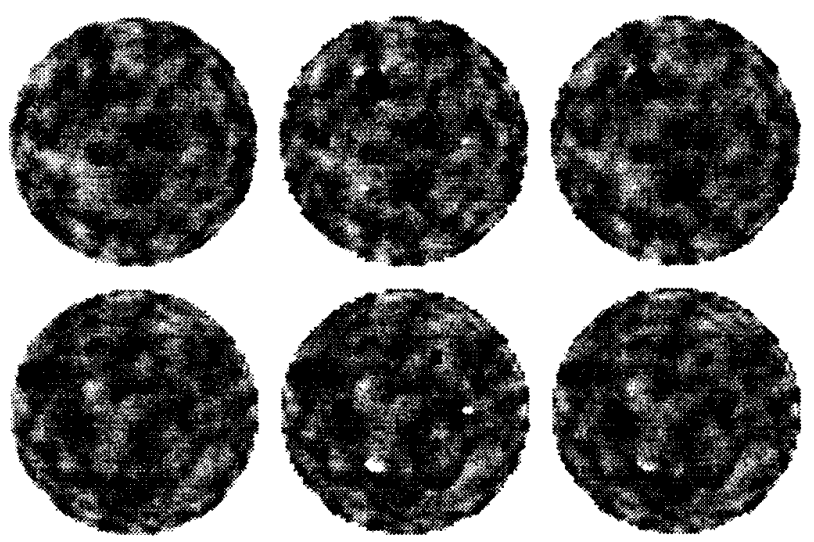

Figure 6. Effect of false labels. Two realizations from simulations with false labels and a uniform emission distribution. The top and bottom rows are independent realizations, while the columns are, from left to right: no labels, binary labels, and blurred labels.

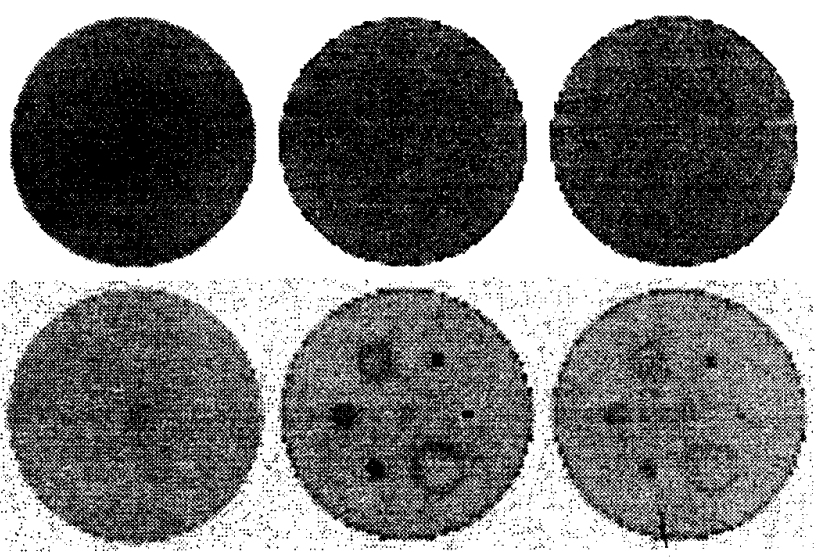

Figure 7. Mean and standard deviation images of the 100 realizations of simulations with false labels. Top row: mean images. Bottom row: standard deviation images. The columns correspond to figure 9 and are, from left to right: no labels, binary labels, and blurred labels.
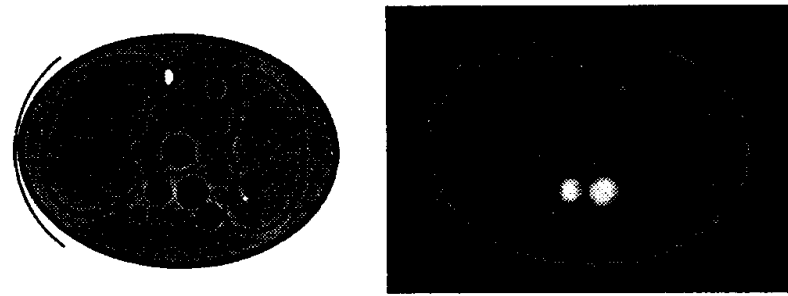

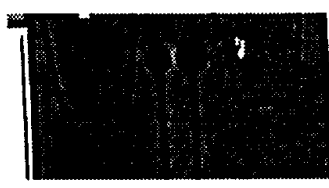

CT

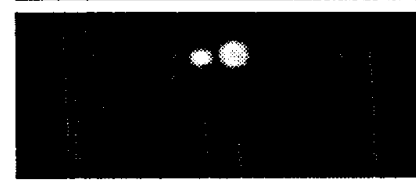

Fused PET/CT
Figure 8. Transverse and frontal sections through the CT and fused PET/CT volume images of the torso phantom, showing the location of the seven contrast spheres (one is in the right lung region). The object at the top of the $C T$ transverse section is a small post taped to the inside of the phantom.

\section{B. EXPERIMENTAL STUDIES}

The alignment accuracy is illustrated by figure 8 , which shows transverse and frontal sections through the reconstructed CT volume image and the fused PET/CT volume image. Transverse sections through the reconstructed volume PET images are shown in figure 9. Representative images are shown for penalty weights based on the three cases of: no labels, binary labels, and blurred labels.

\section{DISCUSSION AND CONCLUSIONS}

The potential advantages and problems of including anatomical information are apparent in figure 2 . These results are only meant to be illustrative, since the reduction of statistical noise is of equal importance. The use of binary labels is problematic in the case of a functional mismatch. The use of blurred labels greatly reduced, but did not eliminate, the variance in the regions of the false labels. Not surprisingly, the variance images in the cases of accurate and false labels were very similar. 


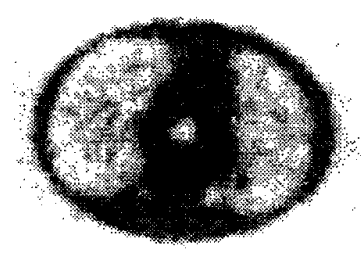

No labels

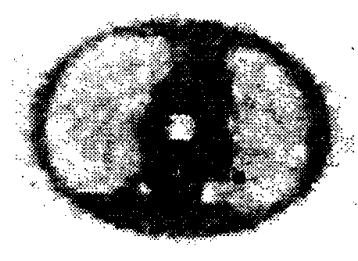

Binary labels

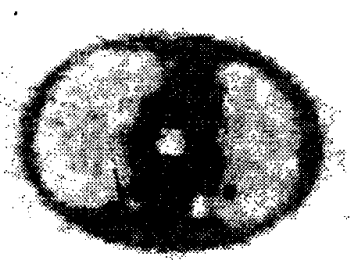

Blurred labels
Figure 9. Transverse sections through reconstructed volume images of the measured 3D phantom showing four hot contrast objects and three cold contrast objects.

In the case where the labels were shifted by $5 \mathrm{~mm}$ (one pixel) from the true emission contrast regions, there was a reduction in image contrast, as shown by comparing the mean images in figures 4 and 6 . In addition there was a shift in the apparent centroid of the objects that corresponded to the shift in the label locations, as shown in figure 7. There was also a significant reduction in the ROI variance. The net effect is shown in figure 8 for two of the objects, which indicates that a $5 \mathrm{~mm}$ shift is sufficient to remove any advantage of using anatomical information. This amount of label misalignment, however, does not necessarily degrade the noise-bias performance relative to PWLS without anatomical information.

For the experimental studies the use of both binary and blurred labels leads to improved contrast for the hot and cold contrast objects of all sizes. It should be noted, however, that both the experimental and simulated phantoms are piecewise continuous objects, and are ideally suited for this type of reconstruction method. One drawback of this method is the possibility of over-smoothing images, which may lead to increased false positive and/or false negative detection rates.

In summary, the SMART tomograph, by acquiring aligned PET and CT data, allows the inclusion of anatomical information to guide the reconstruction of whole-body PET data. In the case of accurate PET-CT alignment, the use of blurred anatomical labels improves the bias-variance tradeoff relative to not using labels. In the case of a PET-CT functional mismatch, blurred labels can reduce the substantial variance introduced by binary penalty weights. Further work is needed, however, to determine the net impact of this approach on diagnostic utility.

\section{ACKNOWLEDGMENTS}

We thank David Manthei and Daniel Holt for their assistance in preparing the phantom experiments.

\section{REFERENCES}

[1] Zhou Z, Leahy RM, et al., "A comparative study of the effects of using anatomical priors in PET reconstruction," Proccedings of: The 1993 IEEE Nuclear Science Symposium and Medical Imaging Conference, pp. 17491753, 1994.

[2] Townsend DW, Beyer T, et al., "The SMART Scanner: A Combined PET/CT Tomograph for Clinical Oncology," Proccedings of: IEEE Nuclear Science Symposium and Medical Imaging Conference, Toronto, Ontario, pp. CDROM, 1998.

[3] Kinahan PE, Michel C, et al., "Fast Iterative Image Reconstruction of 3D PET Data," Proccedings of: IEEE Nuclear Science Symposium and Medical Imaging Conference, Anaheim, CA, pp. 1918-1922, 1996.

[4] Defrise M, Kinahan PE, et al., "Exact and Approximate Rebinning Algorithms For 3-D Pet Data," IEEE Trans Med Imag, Vol. 16, pp. 145-158, 1997.

[5] Hudson $H$ and Larkin $R$, "Accelerated image reconstruction using ordered subsets of projection data," IEEE Trans Med Imag, Vol. 13, pp. 601-609, 1994.

[6] Fessler JA, "Penalized weighted least squares image reconstruction for positron emission tomography," IEEE Trans Med Imag, Vol. 13, pp. 290-300, 1994.

[7] Comtat C, Kinahan PE, et al., "Fast Reconstruction of 3D PET Data with Accurate Statistical Modeling," IEEE Trans Nuc Sci, Vol. 45, pp. 1083-1089, 1998.

[8] Ouyang X, Wong WH, et al., "Incorporation of correlated structural images in PET image reconstruction.," IEEE Trans Med Imag, Vol. 13, pp. 627-64-, 1994.

[9] Fessler JA, Clinthorne NH, et al., "Regularized emission image reconstruction using imperfect side information," IEEE Trans Nuc Sci, Vol. 39, pp. 1464-1471, 1992. 